NEWS

\title{
When good cholesterol turns bad
}

Last week's dramatic failure of a drug trial aimed at boosting 'good' cholesterol is bad news for Pfizer, the world's largest drug company. But will it also dash the hopes of those looking for a new way of preventing heart attacks?

Until now, the cholesterol story has been portrayed as a simple one, with just two characters: 'bad' cholesterol, which promotes heart disease, and 'good" cholesterol, which protects against it. Driving down levels of the former helps the heart, as should boosting levels of the latter.

The first idea is broadly accepted, thanks to statins, the best-selling drugs that cut levels of bad cholesterol (more properly called lowdensity lipoproteins, or LDLs). But with Pfizer being obliged on 2 December to abandon trials of its drug candidate torcetrapib, which was designed to increase levels of good cholesterol, the second idea has suffered a serious setback.

The trial in 15,000 people was called off because of the increased number of people who had died after taking the drug and a statin for 18-20 months, compared with a group taking the statin alone; 82 people died on the combined therapy, compared with 51 on the statin. ${ }^{\alpha}$ This cancellation came as a complete shock," says John Chapman, who has studied torcetrapib at the biomedical research institute INSERM in Paris.

But not everyone was so surprised. Some specialists have been harbouring doubts that

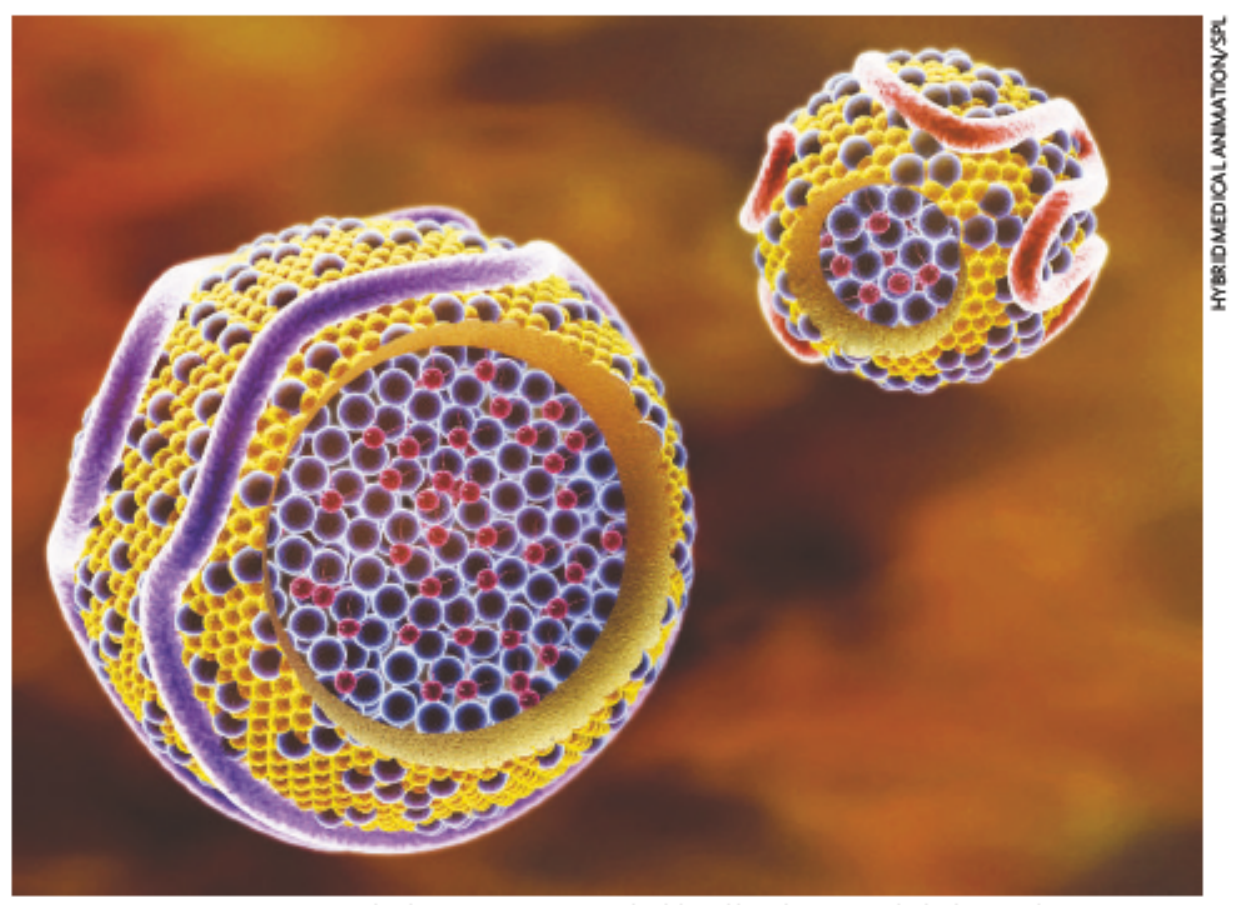

Some experts are questioning the benefits of rais ing the blood levels of 'good' choles terol.

certain ways of raising good cholesterol high-density lipoproteins, or HDLs - might cause more problems than they solve.

Bumping up HDL levels makes sense on the basis of many epidemiological studies showing that people with low levels of HDLs tend to be at higher risk of heart disease. But Pfizer's trial was one of the first to test whether this beneficial effect could be mimicked using a drug to specifically boost levels of HDLs.

HDLs labour away in the bloodstream like a huge fleet of garbage trucks, carting off cholesterol to the liver for safe disposal. LDLs do the opposite, dumping their cholesterol load

\section{Changes at the helm fuel Salk expansion plans}

\section{SAN DIEGO}

The prestigious Salk Institute for Biological Studies is on the brink of change. Fresh leadership at the California research facility in La Jolla is set to direct it towards a major expansion.

The search for a president has already begun after neuroscientist Richard Murphy announced on 8 December that he will leavenext July, after nearly seven years in the post.

And Irwin Jacobs, an electrical engineer who led his company, San Diego-based Qualcomm, to pre-eminence in mobile-phone technology, assumed the nine-year chairmanship of the Salk board lastmonth. Billionaire Jacobs is a popular choice. He has a stellar international business reputation, a track record in philanthropy, and is the firstboard chair to come from the Salk's home town.

"These developments create the opportunity for the Salk to unlock its substantial potential," says Kenneth Chien, a Harvard physician who has a Salk adjunct faculty position. "This is a very opportune moment tocapitalize ona sciencedriven agenda."

Although ithas only 62 faculty members, the Salk has set a sterling recordfor answering some of the most basic questions in biology, and its strength and direction are closely watched.

The leadership changes come as the institute prepares to complete an expansion firstenvisaged in the 1960 s, when polio researcher Jonas Salk founded the institute. Whereas three other non-profit research institutes in San Diego's cluster recently accepted lavish financial payouts to openlabs in Florida, Salk officials spurned its suitors (see Nature 442, 729; 2006). "Three or four Florida groups asked us to consider moving," says Murphy. "We were not interested. Our strength is remaining small and interactive." The Salk plancalls for a new building for labs; a research facility for photonics, mass spectroscopy and humanstem-cell projects; a child-care centre; and ocean-view residences for visiting scientists. Salk officials hope to win the necessary government approvals by next June, while launching a funding drive for the project. Costs are estimated to exceed $\$ 100$ million.

The institute is considered a national architecturallandmark - it was designed by Louis Kahn and crowns an ocean-front cliff.So the expansion is already drawing 
in blood vessels where it can accumulate and potentially dog the vessels. Torcetrapib works by inhibiting a protein called cholesteryl ester transfer protein (CETP), which moves cholesterol from HDLs to LDLs. This, it was hoped, would help clear more cholesterol from the bloodstream.

As Wall Street frets over the implications of Pfizer's failed trial (see page 805 ), cardiovascular specialists are equally abuzz about why the failure occurred. They want to know whether the problem lies with this drug alone, whether all drugs that inhibit CETP might be prone to similar problems, or whether meddling with HDL levels is inherently dangerous. The last possibility would have serious repercussions for researchers and drug companies already investing heavily in an array of other methods to boost or alter HDL levels.

The reason for the increased deaths in the trial participants will not become clear until the results of the abandoned trial are released. One hint lies in evidence from earlier studies that torcetrapib raises blood pressure in some people. That might have proved fatal in patients with already raised blood pressure. If this is the case - or if the problems are caused by some other factor unique to torcetrapib - then other drugs being developed to inhibit CETP might not suffer the same downfall. One drug being developed by Roche, for example, is thought to interact with a different part of the CETP molecule and might not affect blood pressure.

But some researchers are asking whether the very concept of inhibiting CETP is flawed. Normally, when the HDL trucks dump their cholesterol at the liver, they do so by offloading some of it to LDLs on the way. But CETP partially prevents the transfer of cholesterol from HDLs to LDLs, so there has been some debate about whether drugs such as torcetrapib could cause HDLs to accumulate large amounts of cholesterol that they cannot traffic out of the body normally. "You're blocking a physiological mechanism to eliminate cholesterol," says Moti Kashyap, who directs atherosclerosis research at the VA Medical Center in Long Beach, California.

According to other experts, the idea of boosting good cholesterol may be far too simplistic to start with. HDLs are complicated partides, they point out, composed of different proteins and lipids. Each particle's components and chemical modifications are thought to affect how it behaves, and some recent studies have suggested that certain variants of HDLs may actually promote cardiovascular disease.

In one such study, a team led by Ben Ansell at the University of California, Los Angeles,
"One hint lies in evidence that torcetrapib raises blood pressure in some people."
Following this line of thinking, HDLs themselves should be reclassified into 'good' and 'bad' forms. This means that total HDL levels could be a poor measure of its actions and that heart drugs would work only if they boost the correct form of HDL. "It might not be enough to have a lot of HDL - what matters is how that HDL functions," says Prediman Shah, director of cardiology and atherosclerosis at the CedarsSinai Medical Center in Los Angeles.

Some researchers are optimistic about efforts to boost one of these particularly 'good' breeds, a variant of a protein found in HDL. The protein, called ApoA-1 Milano, was found in residents of a rural Italian village who have very low HDL levels but do not seem to be prone to cardiovascular problems. In 2003, a team led by Steven Nissen of the Cleveland Clinic in Ohio showed that five infusions of this protein in humans shrank plaques in the arteries (S. E. Nissen et al. J. Am. Med. Assoc. 290, 2292-2300; 2003). The approach is now being pursued by Pfizer.

Many cholesterol specialists say studied people who defy medical expectation because they have particularly high HDL levels, but still develop heart disease. In lab assays, the team found that HDLs from these people behave in ways that could promote the build-up of cholesterol in the arteries rather than protect against it (B. J. Ansell et al. Circulation 108, 2751-2756; 2003). Similarly, Ansell says, Pfizer's drug might have favoured the formation of damaging or dysfunctional forms of HDL. He adds that his finding is "very analogous to what I'm sure happened in this trial". they are still optimistic that, by one means or another, tweaking HDLs can add to the benefits already accrued by statins. But at least some admit that the case for raising good cholesterol is nowhere near as watertight as that for lowering bad cholesteroL ${ }^{\star}$ There are many ways to raise HDLs. The problem is we really don't know if any of them work [ to prevent the cardiovascular disease]," says Christie Ballantyne, a cardiovascular specialist at Baylor College of Medicine in Houston, Texas.

Helen Pearson fire. A local designorganization has criticized the plan, even though much of the new building will be underground, temporary structures and parking lots will disappear, and no final model has beendecided.

Having lacobs as board chairm an should help considerably, though, especially given his services to the community - his many donations include $\$ 110$ million to the University of California, San Diego, and $\$ 120$ million to the San DiegoSymphony.

"We plan to focus on raising money for the institute's scientific work," says Jacobs. The number of faculty to be added with the expansion has not been decided, but a $10 \%$ increase has beensuggested.
Murphy's tenure had been a fruitful one. During his term, 16 faculty members were hired, major research centres were created, about a third of the labs were renovated and the endowment rose. "It's been a great six years," says Murphy. "I thought it best to go before the institute started the capital campaign."

Looking to the future, faculty head Inder Verma says: "For the first time, we have a board chairman who is from San Diego; this is very positive. He is a community leader with academic credentials." On the presidential search, Verma notes: "The faculty would prefer someone from outside, to bring in new blood." Rex Dalton

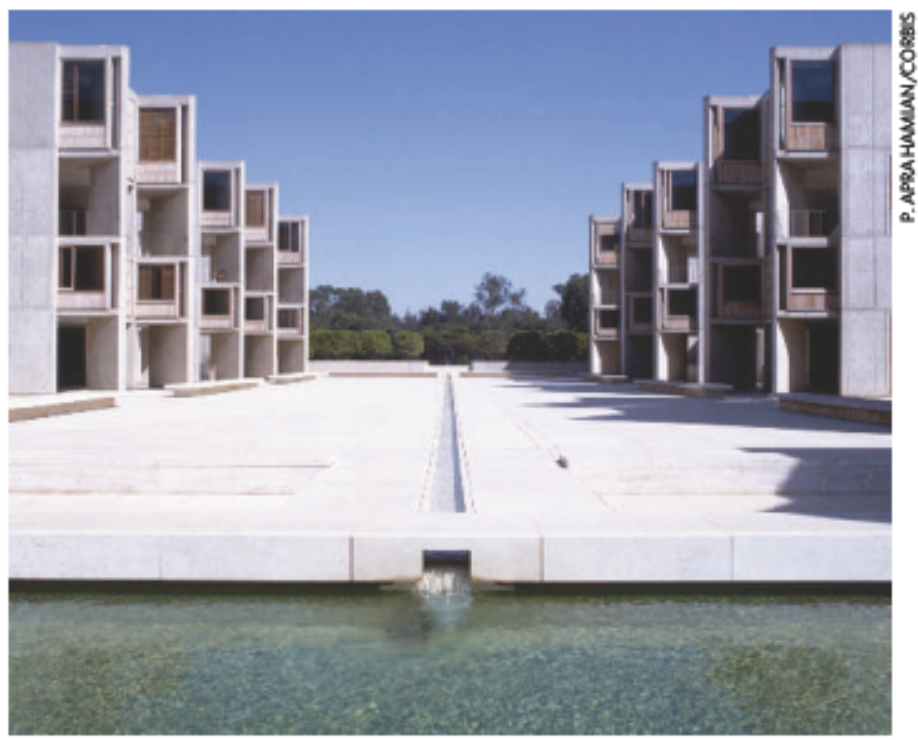

Anarchitecturallandmark, the Salk Institute is set to grow. 